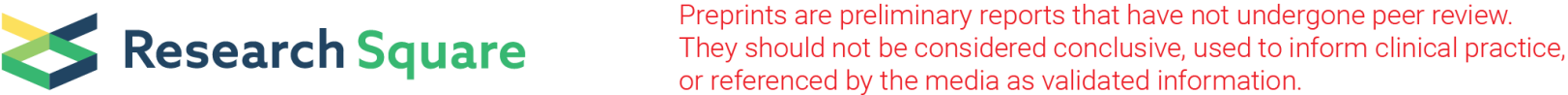

\section{Intestinal Dysbacteriosis-Propelled T Helper 17 Cells Activation Mediate the Perioperative Neurocognitive Disorder Induced by Anesthesia/Surgery in Aged Rats}

Jie Sun ( $\nabla$ dgsunjie@hotmail.com )

Southeast University Zhongda Hospital https://orcid.org/0000-0002-8647-7867

\section{Y.Z. Wen}

Southeast University Zhongda Hospital

\section{S.W. Feng}

Nanjing Medical University Affiliated Healthcare Hospital for Women and Infants: Nanjing Maternity and Child Health Care Hospital

\section{H.Y. Dai}

Nanjing Medical University

\section{Mao}

Nanjing Medical University

\section{Z.H. Zhou}

$$
\text { Nanjing Medical University }
$$

B. Li

Southeast University Zhongda Hospital

\section{C.R. Wang}

Nanjing Medical University

X.C. Cai

Nanjing Medical University

S.M. Li

Southeast University Zhongda Hospital

\section{J.J. Yang}

Southeast University Zhongda Hospital

Q. Ren

Southeast University Zhongda Hospital

\section{Research}

Keywords: Th17, IL17, microglia, PND, intestinal flora 
Posted Date: September 21st, 2021

DOl: https://doi.org/10.21203/rs.3.rs-880055/v1

License: (c) (i) This work is licensed under a Creative Commons Attribution 4.0 International License. Read Full License 


\section{Abstract}

Background: Perioperative neurocognitive disorders (PND) occur frequently in elderly patients after surgery, but the mechanism of PND is not very clear at present. It is reported that anesthesia/surgery could cause intestinal flora imbalance and induce neurocognitive impairment. However, the effect of intestinal flora on PND is poorly understood. We previously found that peripheral interleukin-17A (IL17A) destroyed the blood-brain barrier (BBB), leading to central inflammation and neurocognitive impairment. The small intestine is the main place where Th17 cells are produced. Therefore, we hypothesized that Th17 cells and IL-17 may be an important bridge for intestinal microbes to cause neuroinflammation.

Methods: Exploratory laparotomy was performed to establish PND model under sevoflurane anesthesia. 16S rRNA high-throughput sequencing was used to detect the changes of intestinal flora. To explore the relationship between intestinal flora and PND, compound antibiotics were used to eliminate intestinal flora before anesthesia/surgery, and behavior tests, such as open field, $Y$ maze, and fear conditioning tests were applied to detect the changes of memory ability and which was compared with the rats that did not receive compound antibiotics. The number of Th17 cells and Foxp3 cells was detected by flow cytometry in the Peyer's patches (PP), mesenteric lymph nodes (MLN), blood and brain. Hippocampus IL17, IL17RA, IL6 and IL10 were detected by Western blot. Hippocampus IL17, IL17R and IBA1 (ionized calcium binding adaptor molecule1) were detected by immunofluorescence.

Results: Anesthesia/surgery caused intestinal flora imbalance and induced neurocognitive impairment, increased the number of Th17 cells in the PP, MLN, blood and brain, up-regulated the lever of IL17, IL17R and inflammatory factor production in the hippocampus. The administration of compound antibiotics before anesthesia/surgery evidently inhibited this effect, including decreased the number of Th17 cells, down-regulated the lever of IL17, IL17R and inflammatory factor production, and improved the memory function. In addition, we found that IL17R was co-labeled with IBA1 in a large amount in the hippocampus through immunofluorescence double-staining.

Conclusion: Our study suggested that intestinal dysbacteriosis-propelled T helper 17 cells activation might play an important role in the pathogenesis of PND.

\section{Introduction}

Perioperative neurocognitive disorder (PND) is an overarching term that is used for cognitive impairment in the perioperative period [1], which is characterized as changed neurocognitive function, such as awareness, memory, attention, information processing, and cognitive flexibility [2]. PND often occurs in elderly patients, for days, months or even years $[3,4]$. Due to different types of surgery and research methods, the incidence of PND varies between $8.9 \%$ and $46.1 \%[4,5]$. PND leads to delayed postoperative recovery, decreased self-care ability, increased hospitalization time and medical expenses, and brings heavy burden to the family and society [6]. 
The mechanism of PND is not very clear at present. The central immune inflammatory response is one of the widely accepted theory. The key point of this theory is that surgical trauma causes peripheral inflammation, and immune messages are transmitted to the brain, triggering neuroinflammation and leading to cognitive dysfunction. The previous experiments of our research group found that tibial fractures caused significantly up-regulation of IL-17A in the serum and hippocampus of elderly mice. Peripheral administration of IL-17A antibody reduced the level of IL-17A in the hippocampus after surgery, reduced blood-brain barrier dysfunction and inflammation in the hippocampus, and improved postoperative cognitive impairment [7]. Approximately $70 \%$ of the immune cells are present in the intestine. The interaction between gut microbes and the host is very important in the development and maintenance of the host immune system [8]. Intestinal microbes are an important factor affecting the proliferation and differentiation of Th17 cells [9].

In recent years, the role of intestinal flora in various diseases has attracted widespread attention. There have been a large number of documents confirmed that intestinal flora was involved in Alzheimer's disease (AD) [10], Parkinson's disease (PD) [11], stroke [12], Amyotrophic lateral sclerosis (ALS) [13], autism [14], postoperative cognitive dysfunction (POCD) [15] and other diseases. Studies have confirmed that anesthesia/surgery affected the abundance and diversity of intestinal flora in mice [15-17]. Probiotics or antibiotics administrated before anesthesia/surgery to regulate intestinal flora could improve the memory ability of old mice [15]. A clinical study also reported that administration of probiotics during the perioperative period significantly reduced the incidence of POCD [18]. The above studies have shown that the intestinal flora is inextricably linked with PND, but how the intestinal flora acts on the CNS remains unclear.

The role of Th17 cells in the microbe-gut-brain axis has attracted more and more attention. High-salt diet induced the activation of Th17 cells in the intestine, increased the secretion of IL 17A in circulation, and then damaged vascular endothelial cells, resulting in decreased cerebral perfusion and cognitive dysfunction [19]. In addition, researchers have detected IL-17 + $\delta \delta$ T cells from the intestines in the meningeal lymphatics of stroke model mice [12]. Another study found that virus infection in pregnant mouse caused Th17 cells to activate and secrete IL-17 cytokines. These molecules bound to the receptors in the brain of the developing embryo through the placenta, and the offspring exhibited autism-like behavior [20]. The above studies suggested that Th17 cells and IL-17 may be an important bridge for intestinal microbes to influence CNS.

We hypothesize that anesthesia/surgery leads to intestinal flora imbalance, activates the intestinal immune system, and increases the activation of intestinal Th17 cells and the secretion of IL17. Th17 cells and IL17 act on hippocampal microglia, promote central inflammation and mediate PND.

\section{Materials And Methods \\ 2.1 Animals}


The animals used in this study were 15-month-old SPF male SD rats, which were purchased from SiPeiFu (Beijing) Biotechnology Co., Ltd. The rats were raised in the SPF animal room of Southeast University. The animal room was 12-hour day/night, the room temperature was $24 \pm 1^{\circ} \mathrm{C}$, and the relative humidity was $45 \%$. Animals had free access to food and water. The protocols of this study were approved by the Ethics Committee of Zhongda Hospital, School of Medicine, Southeast University, Nanjing, Jiangsu, China (permit number: 20200701006). All procedures of our study followed the guideline of the National Institutes of Health guide for the care and use of Laboratory animals (NIH Publications No. 8023, revised 1978).

\subsection{Experiment grouping}

48 rats were randomly divided into 4 groups: control group (CON), compound antibiotics group (ANT), anesthesia/surgery group (SUR), and compound antibiotics + anesthesia/surgery group (ANT + SUR). According to Barrientos' method [21], exploratory laparotomy was performed under sevoflurane anesthesia with minor modifications. After successful induction of anesthesia, we shaved the abdominal area and disinfected the surgical area with iodophor. Then we made a midline incision (about $3 \mathrm{~cm}$ long, and penetrating the peritoneal cavity) and explored the internal organs, intestines and muscle tissues. Finally, sterile 3 - 0 chrome-plated gut sutures were used to suture the peritoneum, abdominal muscles and skin in layers. The total duration of the entire operation was 30 minutes. For rats in the CON group, neither anesthesia nor surgery was performed. From day 1 to day 6 after anesthesia/surgery, rats feces were collected every day, and the weight of the rats was measured and recorded. The fecal samples were stored in a refrigerator at $-80^{\circ} \mathrm{C}$.

\subsection{Antibiotics treatment}

Compound antibiotics were used to relatively eliminate the intestinal flora of rats in ANT group and ANT + SUR group 4 weeks before anesthesia/surgery. The compound antibiotics (vancomycin $(500 \mathrm{mg} / \mathrm{L})$, neomycin $(1 \mathrm{~g} / \mathrm{L})$, ampicillin sodium $(1 \mathrm{~g} / \mathrm{L})$, and metronidazole $(1 \mathrm{~g} / \mathrm{L}))$ were prepared with sterile water according to the required concentration, and were used by free-drinking. This dosing schedule is consistent with multiple studies for eliminating gut microbiome [22]. The water consumption was monitored during the 4-week treatment period, and the water consumption was equivalent to plain water. The intestinal flora in rats' feces was detected by gene sequencing to determine whether the intestinal flora had been eliminated. The exploratory laparotomy was performed in ANT + SUR group after antibiotics treatment.

\subsection{Open field test (OFT)}

On the third day after anesthesia/surgery, open field test was performed to evaluate the rats' exploratory movement. Put each rat gently in the center of the topless black plastic test chamber $(100 \mathrm{~cm} \times 100 \mathrm{~cm} x$ 40cm; XR-XZ301; Shanghai Softmaze Information Technology Co., Ltd (Shanghai, China)) and let it explore independently for 5 minutes. The computer system recorded the movement trajectory of the rats. Before and after each rat was tested, the bottom and four walls of the test chamber were sprayed with 
$75 \%$ alcohol to avoid the residual odor or urine and feces of the previous rat from affecting the exploratory behavior of the next rat.

\subsection{Y maze test}

The $Y$ maze test was performed to detect working memory of rats 2 hours after open field test. The $Y$ maze (XR-XZ1032; Shanghai Softmaze Information Technology Co., Ltd (Shanghai, China)) consists of three identical arms $(50 \mathrm{~cm} \times 10 \mathrm{~cm} \times 30 \mathrm{~cm})$ and a connecting area, and the angle between the three arms is 120 degrees. Put each rat gently at the end of any arm of the $Y$ maze and allow it to explore the maze for 8 minutes. The camera system recorded the rats' movement trajectory. According to the movement trajectory, the following data were obtained: (1) Total number of arm access; (2) Maximum number of alternations: total number of arm access subtract 2; (3) Actual number of alternations: entering three arms one after the other, such as 1,2,3 or 1,3,2. Spontaneous alternation ratio = actual alternation times/maximum alternation times $\times 100 \%$. After each rat was tested, $75 \%$ alcohol was sprayed to wipe the inner wall and bottom of the $Y$ maze.

\subsection{Fear conditioning test (FC)}

To test the spatial memory ability, the rats were trained and tested for fear conditioning. Put each rat into a conditioning chamber $(30 \mathrm{~cm} \times 30 \mathrm{~cm} \times 45 \mathrm{~cm}$; XR-XC404; Shanghai Softmaze Information Technology Co., Ltd (Shanghai, China)) and allowed it to explore the chamber for 3 minutes. Then a 30-second tone ( $76 \mathrm{~dB}, 3 \mathrm{kHz})$ stimulation was administered, followed by a 2-second foot shock (1.5 mA). Each rat stayed in the conditioning chamber for 30 seconds after foot shock, and then returned to its previous cage. 24 hours later, the contextual fear conditioning test was performed (a hippocampus-dependent task). Each rat was placed in the same conditioning chamber where it was trained before, and observed for 5 minutes without tone stimulation or foot shock. Recorded the rats' freezing time. Freezing definition: There was no visible movement except breathing. Before and after the test of each rat, $75 \%$ alcohol was sprayed to clean the bottom and surroundings of the test box.

\subsection{Western blot}

On the sixth day after the anesthesia/surgery, one side of the hippocampal tissue was harvested and placed in tissue lysis solution (RIPA: PMSF = 100:1) and was ground with a glass grinder. After being thoroughly ground, lysed for 30 minutes. Centrifuged for 20 minutes at $4^{\circ} \mathrm{C}, 12000 \mathrm{rpm}$, and took the supernatant. After BCA quantification, SDS-PAGE was used for protein electrophoresis, and the protein was transferred to PVDF membrane after electrophoresis. After blocking with $5 \%$ calf serum at room temperature for 2 hours, the polyvinylidene fluoride (PVDF) membranes were incubated in the primary antibody (IL17 (Santa-Cruz, \#sc-374218, 1:100), IL17RA (Abcam, \#ab180904, 1:1000), IL6 (Affinity Biosciences, \#DF6087, 1:1000), IL10 (Proteintech, \#20850-1-AP,1:500), $\beta$-Tubulin (Proteintech, \#10068-1$A P, 1: 5000)$ ) at $4^{\circ} \mathrm{C}$ overnight. About 24 hours later, the PVDF membrane was rinsed with Tris Buffered Saline with Tween (TBST). Then the PVDF membrane were incubated in the secondary antibody (goat anti-mouse IgG (Servicebio, \#GB23310, 1:5000), goat anti-rabbit IgG (Servicebio, \#GB23303, 1:5000)) at room temperature for 2 hours. Then the PVDF membrane was rinsed with TBST. Spread the ECL 
luminescent liquid evenly on the PVDF membrane and waited for exposure. Image analysis was performed using Image $\mathrm{J}$ software (National Institutes of Health, Bethesda, MD, USA).

\subsection{Immunofluorescent staining}

On the sixth day after anesthesia/surgery, the rats were deeply anesthetized with sevoflurane, and were perfused with $0.9 \%$ sodium chloride and $4 \%$ paraformaldehyde (PFA) respectively. Each intact brain tissue was carefully taken out, and be placed in pre-cooled $4{ }^{\circ} \mathrm{C} 4 \%$ PFA for $12-16$ hours. Dehydrated brain tissue with $30 \%$ sucrose. When each brain tissue sunk to the bottom of sucrose, it was embedded in OCT and stored in a refrigerator at $-80^{\circ} \mathrm{C}$. Coronal slices of brain tissue, $30 \mu \mathrm{m}$ in thickness, were laid flat on glass slides. The sections were washed with phosphate buffered saline (PBS; pH 7.4) and phosphate buffered saline with Triton (PBST), and blocked with 10\% goat serum at room temperature for 2 hours. After blocking, the sections were incubated in the primary antibody (IL17 (Santa-Cruz, \#sc-374218, 1:100), IL17R (Santa-Cruz, \#sc-376374, 1:50), IBA1 (Wako, \#019-19741, 1:500)) at $4^{\circ} \mathrm{C}$ overnight. 24 hours later, washed the sections with PBST. Sections were incubated with the secondary antibody (Alexa Fluor 488 goat anti-rabbit (Proteintech, \#SA00013-2, 1:500), Alexa Fluor 488 goat anti-mouse (Proteintech, \#SA00013-1, 1:500), Alexa Fluor 647 goat anti-mouse (Invitrogen, \#A-21235, 1:1000)) at room temperature for 2 hours. Washed the sections with PBST and PBS and incubated with 4',6-diamidino-2phenylindole (DAPI) for nuclear staining. The fluorescence image was captured with a confocal microscope (Olympus, Japan). Image analysis was performed using Image J software (National Institutes of Health, Bethesda, MD, USA).

\subsection{S rRNA high-throughput sequencing}

Fecal samples from the third day after anesthesia/surgery were used for 16S rRNA high-throughput sequencing. PowerMax DNA extraction kit (MoBio Laboratories, USA) was used to extract DNA from rats' fecal samples. The DNA content and the quality of DNA in the extracted samples were measured and evaluated by NanoDrop ND-1000 spectrophotometer and Agarose gel electrophoresis independently.

We used forward primer 515F (5'-GTGCCAGCMGCCGCGGTAA - 3') and reverse primer 806R (5'GGACTACHVGGGTWTCTAAT-3') to PCR amplify the V4 region of the bacterial 16S rRNA gene. PCR reaction system: $25 \mu$ of high-fidelity enzyme (Phusion High-Fidelity PCR Master Mix with HF Buffer), $3 \mu l$ each of F/R primers, $10 \mu \mathrm{l}$ of DNA template, $9 \mu \mathrm{l}$ of ddH2O. PCR amplification was performed according to the following reaction conditions: pre-denaturation at $98^{\circ} \mathrm{C}$ for 30 seconds; The next 25 cycles:

denaturation at $98^{\circ} \mathrm{C}$ for 15 seconds, annealing at $58^{\circ} \mathrm{C}$ for 15 seconds, extension at $72^{\circ} \mathrm{C}$ for 15 seconds; The final extension is $72^{\circ} \mathrm{C}$ for 1 minute. The AMPure XP Beads (Beckman Coulter, IN) and PicoGreen dsDNA Assay Kit (Invitrogen, USA) were used to purify and quantify the PCR products. After quantification, Illlumina Novaseq 6000 pair-end 2×150 bp platform was used for sequencing at GUHE Info technology Co., Ltd (Hangzhou, China).

QIIME was used to process the raw data. Remove low-quality sequences through the following criteria: sequences that had a length of $<150 \mathrm{bp}$, sequences that had average Phred scores of $<20$, sequences that contained ambiguous bases, and sequences that contained mononucleotide repeats of $>8$ bp. 
Vsearch v1.11.1 was used to pick operational taxonomic units (OTUs) (clustering with 97\% similarity). VSEARCH was used to search for representative sequences set in the SILVA128 database to generate the OTU list, and count the community composition of each sample at each classification level. Remove all OTUs with a content of less than $0.001 \%$ of the total sequence in all samples.

a diversity index was calculated by QIIME software. Beta diversity analysis was performed by using UniFrac distance metrics [23] to investigate the structural variation of microbial communities across samples and visualized via nonmetric multidimensional scaling (NMDS) [24]. The difference in Unifrac distance between groups was compared using the t-test test and the Monte Carlo permutation test. LEfSe default settings and R package "random Forest" default settings were used to compare differences between groups respectively.

\subsection{Cell isolation from PP, MLN and blood}

After each rat was deeply anesthetized with sevoflurane, the PPs and MLN were harvested according to the principle of aseptic operation, and arterial blood was drawn from abdominal aorta. The PPs were placed in $5 \mathrm{ml}$ of collagenase $\mathrm{D}(1 \mathrm{mg} / \mathrm{ml})$ and were incubated in $37^{\circ} \mathrm{C}$ water bath for 30 minutes. Then put the MLN and PPs into a wet 70um sterile cell strainer, and gently ground with the plunger of a $2 \mathrm{ml}$ syringe. Washed the cell strainer with $5 \mathrm{ml}$ RPMI 1640 medium. Centrifuged at $500 \times \mathrm{g}$ for 5 minutes, discarded the supernatant, and washed the cells with RPMI 1640 medium. The whole blood was diluted 1:1 with RPMI 1640 medium, and added it to the upper layer of Ficoll. Centrifuged at $500 \times \mathrm{g}$ for 30 minutes. Collected the peripheral blood mononuclear cells (PBMC) at the junction of the medium and Ficoll into a sterile centrifuge tube. The corresponding flow cytometry antibody was used for staining, and tested with the Attune NxT cytometer (Thermo Fisher Scientific).

\subsection{Isolation of brain leukocytes}

After each brain tissue was harvested from rat, it was chopped into small pieces. Collagenase $D$ $(1 \mathrm{mg} / \mathrm{ml})$ and Dnasel enzyme $(1 \mathrm{mg} / \mathrm{ml})$ were used to digest the tissue and incubated in $37^{\circ} \mathrm{C}$ water bath for 40 minutes. The digested tissue was added into a $70 \mu \mathrm{m}$ sterile cell strainer, a plunger of $2 \mathrm{ml}$ syringe was used to gently grind the remaining tissue mass, and washed the cell strainer with $5 \mathrm{ml}$ RPMI 1640 medium. $100 \%$ Percoll was used to dilute cell suspension to $30 \%$ Percoll cell suspension, and which was slowly added on the top of $5 \mathrm{ml} 70 \%$ Percoll. Without brake, centrifuged at $500 \times \mathrm{g}$ for 30 minutes with horizontal centrifuge. The cell layer between $30 / 70 \%$ density gradient was harvested. The corresponding flow cytometry antibody was used for staining, and tested with the Attune NxT cytometer (Thermo Fisher Scientific).

\subsection{Extracellular staining}

Resuspended the cells with $10 \%$ calf serum and incubated at $4^{\circ} \mathrm{C}$ for 10 minutes. After blocking, extracellular antibodies (LIVE/DEAD ${ }^{\text {TM }}$ Fixable Near-IR Dead Cell Stain Kit (eBioscience, \#L10119), CD3 
PerCP-eFluor-710 (eBioscience, \#46-0030-82), CD45 FITC OX1 (eBioscience, \#11-0461-82), CD4 Super Bright 600 OX35 (eBioscience, \#63-0040-82)) were used to stain cells at $4^{\circ} \mathrm{C}$ for 15 minutes. The cells were washed with sterile PBS, and tested with Attune NxT cytometer (Thermo Fisher Scientific). Image analysis was performed using FlowJo software.

\subsection{Intracellular staining}

Since IL-17 is difficult to stain in the resting state, so the cells need to be activated first. Resuspended the cells in RPMI-1640 medium, which contains 10\% fetal bovine serum, $100 \mathrm{ng} / \mathrm{ml}$ phorbol 12-myristate 13acetate (PMA), $1 \mu \mathrm{g} / \mathrm{ml}$ ionomycin and $3 \mu \mathrm{g} / \mathrm{ml}$ brefeldin for cells activation [12]. Incubated the cells in $37^{\circ} \mathrm{C}, 5 \% \mathrm{CO} 2$ incubator for 4 hours. Centrifuged at $500 \times \mathrm{g}$ for 5 minutes and discarded the supernatant. After blocking with $10 \%$ calf serum and incubated at $4^{\circ} \mathrm{C}$ for 10 minutes, appropriate extracellular antibodies were used for extracellular staining. Then washed the cells, and fixed it with fixation buffer (Foxp3/Transcription Factor Staining Buffer Set (eBioscience, \#00-5523-00)) at $4^{\circ} \mathrm{C}$ for 30 minutes. The cells were washed with permeabilization buffer and incubated with intracellular antibody (IL17 APC eBio 17B7(eBioscience, \#17-7177-81), FOXP3 PE FJK-16s (eBioscience, \#12-5773)) at $4^{\circ} \mathrm{C}$ for 30 minutes. The cells were washed with sterile PBS buffer, and detected with Attune NxT cytometer (Thermo Fisher Scientific). Image analysis was performed using FlowJo software.

\subsection{Statistical analysis}

GraphPad Prism 8.0 software was used for Statistical analysis. Measurement data are expressed as mean \pm standard error (mean \pm SEM). One-way analysis of variance (ANOVA) was used to compare the multiple groups. $\mathrm{P}<0.05$ indicates that the difference is statistically significant.

\section{Results}

\subsection{Anesthesia/surgery changed the composition of intestinal flora in aged rats}

16S rRNA sequencing was performed on fecal samples of aged rats in the CON group and the SUR group. The results showed that the Chao1 index of the SUR group was not significantly different from that of the CON group, while the Shannon and the Simpson index were significantly lower. Detailed data of alpha diversity-related analysis was included in Additional file 1. These results indicated that anesthesia/surgery had a significant impact on the diversity of intestinal flora.

We found that the intestinal flora of the CON group and the SUR group was significantly different at each classification level. At the phylum level, the differences were concentrated in Firmicutes and Proteobacteria. At the family level, there were significant differences in the abundance of Lactobacillaceae, Lachnospiraceae, Turicibacteraceae, Erysipelotrichaceae and Enterobacteriaceae. At 
the genus level, there were significant differences in the abundance of Lactobacillus, Blautia, Turicibacter and Dorea. More detailed data on the composition of intestinal flora between CON group and SUR group was included in Additional file 2. These results suggested that anesthesia/surgery changed the abundance of intestinal flora in aged rats. This is consistent with the previous study of others $[15,16]$.

Figure $1 \mathrm{~A}$ shows that the composition of intestinal flora in the SUR group is significantly different from that of the CON group. The distribution of the intestinal flora in the CON group is relatively scattered and the similarity is poor, while the distribution of the intestinal flora in the SUR group is relatively concentrated and the similarity is good. This indicated that anesthesia/surgery seems to be able to change the composition of intestinal flora in a certain direction. Figure 1B shows the effect of anesthesia/surgery on the relative abundance of the intestinal flora at genus level (top 10). Anesthesia/surgery reduced the abundance of Lactobacillus and increased the abundance of Blautia, Turicibacter and Dorea (Fig. 1C).

In the LEfSe analysis (Fig. 1D), different colors in the figure represent the different species between the CON group and SUR group. There were significant differences in the composition of intestinal flora between the two groups. Compared with the CON group, the SUR group had significant changes in the average and median of the relative abundance of intestinal flora and LDA scores at each classification level of gut bacterial (Fig. 1D-1F). In summary, anesthesia/surgery had a significant impact on the composition of intestinal flora in aged rats.

\subsection{Compound antibiotics improved postoperative cognitive function in aged rats}

To explore the relationship between intestinal flora and PND, compound antibiotics were used to eliminate the intestinal flora before anesthesia/surgery, and observed the changes of cognitive function after anesthesia/surgery. The administration of compound antibiotics before anesthesia/surgery eliminated most of intestinal flora (Additional file 3).

Figure 2A exhibits the behavioral tests flow chart. There were no significant differences in motor function among 4 groups on the third day after anesthesia/surgery $(P>0.05$, Fig. $2 B)$. The correct alternation ratio was significantly decreased in the SUR group than the CON group, while the administration of compound antibiotics evidently increased the correct alternation ratio $(P<0.05$, Fig. $2 C)$. In the FC training phase, there was no significant difference in the freezing time of the 4 groups $(P>0.05$, Fig. 2D). In the FC test phase, the freezing time of rats in the SUR group was significantly reduced compared with the CON group, and rescued in the ANT + SUR group $(P<0.05$, Fig. 2E). The results suggested that anesthesia/surgery may impair the cognitive function of aged rats to some extent, and the administration of compound antibiotics seems to improve the impaired cognitive function. This is consistent with the previous study of others [15].

\subsection{Intestinal flora changed the number of Th17 cells in the periphery and CNS}


The small intestine is the main place where Th17 cells are produced, and the production of Th17 cells is mainly affected by the local microenvironment [9]. Therefore, we used flow cytometry to detect the number of Th17 cells and Foxp3 cells in the PP, MLN, blood and brain. Figure 3A-3D is the flow cytometry representative diagrams of Th17 cells in the brain, MLN and PP of the four groups, and Fig. $3 \mathrm{E}-3 \mathrm{H}$ is the quantification of Th17 cells in the brain, MLN and PP. The number of Th17 cells was significantly increased in the SUR group compared with the CON group, while the administration of compound antibiotics evidently decreased the number of Th17 cells. There was no significant difference in the number of Foxp3 cells among the four groups $(P>0.05$, Fig. 3H- 3K). The above results indicated that anesthesia/surgery induced a significantly increase in the number of Th17 cells in the PP, MLN, blood and brain, while the administration of compound antibiotics reduced the number of Th17 cells.

\subsection{Effects of intestinal flora on the expressions of IL17, IL17RA, IL6, and IL10 in the hippocampus of rats}

The previous study of our research group found that the tibial fractures caused significant up-regulation of IL-17A in serum and hippocampus of aged mice [7]. To explore the effect of intestinal flora on the expression of inflammatory cytokines in the hippocampus of aged rats, we detected IL17, IL17RA, IL6, and IL10 in the hippocampus through western blot. Compared with the CON group, the expression of IL17, IL6 and IL17RA in the hippocampus of the SUR group was significantly increased, while the administration of compound antibiotics evidently decreased the expression of IL17, IL6 and IL17RA (P< 0.05 , Fig. 4A-4F). There was no significant difference in the expression of IL10 in the hippocampus among the 4 groups of rats $(P>0.05$, Fig. $4 G-4 H)$. Full blots for Fig. 4 were included in Additional file 4 . These results suggested that anesthesia/surgery may increase the expression of pro-inflammatory cytokines and their receptors, and the administration of compound antibiotics in advance could attenuate these effects.

\subsection{Effects of intestinal flora on the expression of IL17 in different areas of hippocampus}

We found that the fluorescence intensity of IL17 was significantly increased in the hippocampal CA1, CA3 and DG areas in the SUR group compared with the CON group, while the administration of compound antibiotics evidently decreased the fluorescence intensity of IL17 in the hippocampal CA3 and DG areas $(P<0.05$, Fig. 5A-50), but not the CA1 area $(P>0.05$, Fig. 5E). The results suggested that the anesthesia/surgery significantly increased the expression of IL17 in the hippocampus CA1, CA3 and DG areas of aged rats, and compound antibiotics treatment in advance could attenuate these effects.

\subsection{Effects of intestinal flora on the expression of IL17R and IBA1 in the hippocampus of rats}


We found that IL17R was co-labeled with IBA1 in a large amount in the hippocampus through immunofluorescence double-staining (Fig. 6P-6X). The fluorescence intensity of IBA1 increased significantly in the hippocampal CA1, CA3 and DG areas of the SUR group in comparison with the CON group, while the administration of compound antibiotics evidently decreased the fluorescence intensity of IBA1 $(P<0.05$, Fig. 6A-60). The results suggested that a large amounts of IL17R were expressed with microglia in the hippocampus, considering IBA1 has been widely used to study microglia as its expression is specific [25]. Anesthesia/surgery significantly increased the expression of IBA1 in the CA1, CA3 and DG areas of the hippocampus in aged rats, and compound antibiotics treatment in advance could attenuate these effects.

\section{Discussion}

In this study, we found that anesthesia/surgery caused intestinal flora imbalance and induced neurocognitive impairment in aged rats. Intestinal flora may act on the central nervous system by activating intestinal Th17 cells and increase the secretion of IL17, leading to cognitive impairment, which may be a potential mechanism of PND in aged rats.

There are a huge number of microorganisms on the surface and in the body of our human body, about 10 ^ 14 bacteria, which contain 100 times the number of genomes of humans [26]. The intestinal flora plays an important role in maintaining human health and can affect the CNS in many ways, such as changing the composition of the intestinal flora [27], activating the immune system [28], the vagus nerve [11], the tryptophan metabolites [29], the secretion of intestinal hormones [30], the bacterial metabolites [31] and others. In view of the important role of intestinal flora in maintaining human health, the intestinal flora was even called "neglected human organ" [32].

A large number of studies have confirmed that intestinal flora played an important role in the pathophysiology of neurodegenerative diseases. In the animal model of POCD caused by abdominal exploratory surgery, using the method of cluster analysis, it was found that the abundance and diversity of the intestinal flora in the POCD group was significantly reduced [16]. Similar to the results of the above study, we found that anesthesia/surgery caused intestinal flora imbalance and cognitive impairment in aged rats. When compound antibiotics were given 4 weeks before anesthesia/surgery to relatively eliminate the intestinal flora, the memory ability of old rats was improved. This result is consistent with the study of others [15]. Through 16S rRNA sequencing, we found that the abundance of five family-level intestinal flora changed significantly, including Lactobacillaceae, Lachnospiraceae, Turicibacteraceae, Erysipelotrichaceae and Enterobacteriaceae. At the genus level, the abundance of Lactobacillus, Blautia, Turicibacter and Dorea changed significantly. In patients with depression, multiple sclerosis (MS) and Hashimoto's thyroiditis, the abundance of Blautia and Dorea was increased [33-36]. Lactobacillus regulated emotional behavior and the expression of central gamma-aminobutyric acid (GABA) receptor through the vagus nerve [37]. Lactobacillus reuteri reversed the autism-like behavior of various types of autistic mice [14]. Studies have confirmed that Ruminococcaceae and Lachnospiraceae were related to learning and memory. In the model of memory impairment caused by high-fat diet, the increased 
abundance of Ruminococcaceae and Lachnospiraceae was related to the increase of inflammationrelated genes in the hippocampus [38]. In our study, we found that anesthesia/surgery reduced the abundance of Lactobacillus, and increased the abundance of Lachnospiraceae and Ruminococcaceae in the intestinal flora. Compared with the CON group, the compound antibiotics had no obvious effect on the memory ability of aged rats; Compared with the SUR group, the compound antibiotics improved the memory ability of aged rats. It suggested that the occurrence of PND may correlate to the increase of "harmful" bacteria in the intestine, for example Lachnospiraceae and Ruminococcaceae, and elimination of the "harmful" bacteria through compound antibiotics helped to improve the cognitive function.

Intestinal flora played a decisive role in the maturation of the immune system by stimulating local and systemic immune responses in the intestine [39], especially the maturation of intestinal-associated lymphoid tissues [40]. The lamina propria of the small intestine is rich in Th17 cells, and the changes in the local microenvironment are the main factors for Th17 cells activation. In 2015, Koji Atarashiit et al. discovered that the adhesion of microorganisms to intestinal epithelial cells was a crucial condition for inducing the production of Th17 cells [41]. The level of intestinal Th17 cells could be changed by regulating the intestinal flora through diet $[42,43]$. Some researchers pointed out that intestinal microbes were an important factor affecting the proliferation and differentiation of Th17 cells [9]. The phenotype of differentiation of intestinal CD4 + T cell was determined by microorganisms rather than cytokines [44]. The above studies suggested that the intestinal flora, especially the interaction of intestinal flora with the local microenvironment, was a key factor in the production of Th17 cells.

Anesthetic drugs and surgical trauma may affect the intestinal homeostasis. In our study, we found that anesthesia/surgery caused intestinal flora imbalance, and simultaneously, Th17 cells increased significantly in the PPs, MLN and brain. When compound antibiotics were given before anesthesia/surgery to eliminate the intestinal flora, the number of Th17 cells reduced simultaneously in above tissues. The compound antibiotics treatment significantly reduced intestinal Th17 cells $[45,46]$ and IL-17 cytokines [47].

Studies have shown that IL-17A was involved in the pathophysiological process of neurodegenerative diseases. Researchers found that the level of IL-17A was significantly increased in the cerebrospinal fluid and plasma of patients with MS, $A D$, and PD, and was related to the severity of these diseases $[48,49]$. However, how IL17A causes neurodegenerative diseases is less known yet and controversies remain. Most people believe that IL-17A caused the occurrence and development of diseases by activating glial cells (especially microglia) $[48,50]$. For example, bone cancer pain leaded to the activation of Th17 cells, which promoted the activation of microglia and further aggravated the pain of bone cancer patients. Intrathecal injection of IL17 antibody could relieve pain in such patients [51]. Through immunofluorescence, we found that anesthesia/surgery significantly increased the fluorescence intensity of IL17 and IL17R in the hippocampus of rats. When compound antibiotics were given before anesthesia/surgery, the fluorescence intensity of IL17 and IL17R in the hippocampus was significantly reduced. We also found that most of the IBA1 that specific in the microglia could co-label with IL17R in the hippocampus. These results indicated that anesthesia/surgery caused a significantly increase in the 
number of activated microglia in the hippocampal of aged rats, while the administration of compound antibiotics before anesthesia/surgery could evidently reduce the number of activated microglia.

Microglia are macrophages of the CNS and have multiple functions [52]. Sequencing technology revealed that microglia were the determinants of central nervous system diseases. Through Genome-Wide Association Studies (GWAS), many risk genes for central neurodegenerative diseases (including AD, PD, schizophrenia, autism, and MS) that have been discovered were expressed by microglia [52]. Studies have confirmed that microglia also played an important role in the occurrence of POCD. Study has shown that through inhibiting the colony stimulating factor 1 receptor (CSF1R) in adult mice, it effectively depleted the microglia of the CNS, effectively prevented inflammation infiltration in the hippocampus, and improved cognitive function after tibial fracture [53].

In summary, the intestinal homeostasis of old rats was relatively fragile. Under the combined action of anesthesia and surgery, the intestinal flora was prone to imbalance, harmful flora increased, beneficial flora decreased. Intestinal flora imbalance caused activation and increase of intestinal Th17 cells, which increased the secretion of IL17 and other pro-inflammatory cytokines to aggravate system inflammation response. Increased Th17 cells and IL17 damaged BBB (confirmed in the previous study [7]), entered the CNS and acted on microglia, and consequently caused central immune inflammation and cognitive impairment (Fig. 7).

There are some controversies about the effects of compound antibiotics on cognitive function. Some researchers have found that the use of compound antibiotics to clear the intestinal flora from the weaning of mice affected the new objects recognition memory [54]. Short-term (11 days) use of compound antibiotics affected the new objects recognition memory, but spatial memory was not affected [55]. In our study, we found that the administration of 4-week compound antibiotics has no effect on space and working memory, which is consistent with the previous study [15]. For the effects of compound antibiotics on cognitive function, more research is needed to further confirm.

Our study has some limitations. We did not identify which intestinal flora was involved in Th17 cells activation or cognitive impairment caused by anesthesia/surgery. Further research is needed to identify specific gut bacteria involved in PND, such as transplanting feces or specific gut bacteria into germ-free mice. Besides, in our experiment, we did not set up a simple anesthesia group, because in the process of modeling, our anesthesia time was short to 30 minutes, and a large number of studies also confirmed that sevoflurane has little effect on cognitive function. In primate experiments, it was found that sevoflurane exposure had little effect on the cognitive function of adult monkeys and did not cause the activation of microglia [56]. According to reports, $2.5 \%$ sevoflurane exposure for 1 hour had little effect on the cognitive function in adult rodents [57]. There were even studies reported that sevoflurane could alleviate the cognitive impairment induced by lipopolysaccharide (LPS) [58]. On the other hand, anesthesia and surgery cannot be completely separated. So, we did not set up a simple anesthesia group.

\section{Conclusion}


In summary, our study found that anesthesia/surgery could cause intestinal flora imbalance and cognitive impairment. The intestinal flora may act on hippocampal microglia through intestinal-derived Th17 and its cytokine IL17, leading to PND. Intestinal flora imbalance may be an important pathogenic factor of PND. Our study may provide a new idea and target for the prevention and treatment of PND.

\section{Abbreviations}

AD: Alzheimer's disease; ALS: Amyotrophic lateral sclerosis; BBB: Blood-brain barrier; CNS: Central nervous system; DAPI: 4',6-diamidino-2-phenylindole; EAE: Experimental autoimmune encephalomyelitis; FBS: Foetal bovine serum; FC: Fear conditioning; IBA1: Ionized calcium binding adaptor molecule1; IL-17: Interleukin-17; IL-17A: Interleukin-17A; MLN: Mesenteric lymph nodes; MS: Multiple sclerosis; OFT : Open field test; PD: Parkinson's disease; PBS: Phosphate buffered saline; PBST: Phosphate buffered saline with Triton; PMSF: Phenylmethylsulfonylfluoride; PND: Perioperative neurocognitive disorders; POCD: Postoperative cognitive dysfunction; PVDF: Polyvinylidene fluoride; PP : Peyer's patches; RIPA: Radio Immunoprecipitation Assay; SDS-PAGE: Sodium dodecyl sulfate-polyacrylamide gel electrophoresis; TBST: Tris Buffered Saline with Tween.

\section{Declarations}

\section{Acknowledgements}

We are grateful to the staff in Public Scientific Research Platform of Zhongda Hospital Affiliated to Southeast University for technical assistance.

\section{Funding}

This project was sponsored by the National Natural Science Foundation of China (No.82071196 and No.81801074), the Jiangsu Commission of Health (No. z201949) and the Basic Research Grant of Southeast University.

\section{Availability of data and materials}

The data sets that support the conclusions of this study are included in this manuscript and its supplementary files. All materials used in this study will be provided to researchers confidentially.

\section{Authors' contributions}

YZW, SWF, HYD, MM, ZHZ, BL, CRW, XCC, SML, and JJY performed the experiments. YZW, QR and JS designed the study. $Y Z W$ wrote the manuscript. The final manuscript was read and approved by all authors.

\section{Ethics approval}


The protocols of this study were approved by the Ethics Committee of Zhongda Hospital, School of Medicine, Southeast University, Nanjing, Jiangsu, China (permit number: 20200701006). All procedures of our study followed the guideline of the National Institutes of Health guide for the care and use of Laboratory animals (NIH Publications No. 8023, revised 1978).

\section{Consent for publication}

Not applicable.

\section{Competing interests}

None.

\section{References}

1. Evered, L., et al., Recommendations for the nomenclature of cognitive change associated with anaesthesia and surgery-2018. Br J Anaesth, 2018. 121(5): p. 1005-1012.

2. Fan, W., et al., The Role of Microglia in Perioperative Neurocognitive Disorders. Front Cell Neurosci, 2020. 14: p. 261.

3. Bilotta, F., et al., Postoperative cognitive dysfunction: toward the Alzheimer's disease pathomechanism hypothesis. J Alzheimers Dis, 2010. 22 Suppl 3: p. 81-9.

4. Androsova, G., et al., Biomarkers of postoperative delirium and cognitive dysfunction. Front Aging Neurosci, 2015. 7: p. 112.

5. Monk, T.G., et al., Predictors of cognitive dysfunction after major noncardiac surgery. Anesthesiology, 2008. 108(1): p. 18-30.

6. Nadelson, M.R., R.D. Sanders, and M.S. Avidan, Perioperative cognitive trajectory in adults. Br J Anaesth, 2014. 112(3): p. 440-51.

7. Ni, P., et al., IL-17A contributes to perioperative neurocognitive disorders through blood-brain barrier disruption in aged mice. J Neuroinflammation, 2018. 15(1): p. 332.

8. Logsdon, A.F., et al., Gut reactions: How the blood-brain barrier connects the microbiome and the brain. Exp Biol Med (Maywood), 2018. 243(2): p. 159-165.

9. Calcinotto, A., et al., Microbiota-driven interleukin-17-producing cells and eosinophils synergize to accelerate multiple myeloma progression. Nat Commun, 2018. 9(1): p. 4832.

10. Sun, Y., et al., Intra-gastrointestinal amyloid- $\beta$ 1-42 oligomers perturb enteric function and induce Alzheimer's disease pathology. J Physiol, 2020. 598(19): p. 4209-4223.

11. Kim, S., et al., Transneuronal Propagation of Pathologic a-Synuclein from the Gut to the Brain Models Parkinson's Disease. Neuron, 2019. 103(4): p. 627-641.e7.

12. Benakis, C., et al., Commensal microbiota affects ischemic stroke outcome by regulating intestinal $\gamma \delta$ $T$ cells. Nat Med, 2016. 22(5): p. 516-23. 
13. Blacher, E., et al., Potential roles of gut microbiome and metabolites in modulating ALS in mice. Nature, 2019. 572(7770): p. 474-480.

14. Sgritta, M., et al., Mechanisms Underlying Microbial-Mediated Changes in Social Behavior in Mouse Models of Autism Spectrum Disorder. Neuron, 2019. 101(2): p. 246-259.e6.

15. Jiang, X.L., et al., Intestinal dysbacteriosis mediates the reference memory deficit induced by anaesthesia/surgery in aged mice. Brain Behav Immun, 2019. 80: p. 605-615.

16. Zhan, G., et al., Anesthesia and surgery induce cognitive dysfunction in elderly male mice: the role of gut microbiota. Aging (Albany NY), 2019. 11(6): p. 1778-1790.

17. Yang, X.D., et al., Effects of prebiotic galacto-oligosaccharide on postoperative cognitive dysfunction and neuroinflammation through targeting of the gut-brain axis. BMC Anesthesiol, 2018. 18(1): p. 177.

18. Wang, P., et al., Perioperative probiotic treatment decreased the incidence of postoperative cognitive impairment in elderly patients following non-cardiac surgery: A randomised double-blind and placebo-controlled trial. Clin Nutr, 2021. 40(1): p. 64-71.

19. Faraco, G., et al., Dietary salt promotes neurovascular and cognitive dysfunction through a gutinitiated TH17 response. Nat Neurosci, 2018. 21(2): p. 240-249.

20. Kim, S., et al., Maternal gut bacteria promote neurodevelopmental abnormalities in mouse offspring. Nature, 2017. 549(7673): p. 528-532.

21. Barrientos, R.M., et al., Intracisternal interleukin-1 receptor antagonist prevents postoperative cognitive decline and neuroinflammatory response in aged rats. J Neurosci, 2012. 32(42): p. 146418.

22. Greer, R.L., et al., Akkermansia muciniphila mediates negative effects of IFNY on glucose metabolism. Nat Commun, 2016. 7: p. 13329.

23. Lozupone, C.A., et al., Quantitative and qualitative beta diversity measures lead to different insights into factors that structure microbial communities. Appl Environ Microbiol, 2007. 73(5): p. 1576-85.

24. Ramette, A., Multivariate analyses in microbial ecology. FEMS Microbiol Ecol, 2007. 62(2): p. 142-60.

25. Calcia, M.A., et al., Stress and neuroinflammation: a systematic review of the effects of stress on microglia and the implications for mental illness. Psychopharmacology (Berl), 2016. 233(9): p. 163750.

26. Eckburg, P.B., et al., Diversity of the human intestinal microbial flora. Science, 2005. 308(5728): p. 1635-8.

27. Claesson, M.J., et al., Composition, variability, and temporal stability of the intestinal microbiota of the elderly. Proc Natl Acad Sci U S A, 2011. 108 Suppl 1(Suppl 1): p. 4586-91.

28. Ivanov, II, et al., Induction of intestinal Th17 cells by segmented filamentous bacteria. Cell, 2009. 139(3): p. 485-98.

29. Ruddick, J.P., et al., Tryptophan metabolism in the central nervous system: medical implications. Expert Rev Mol Med, 2006. 8(20): p. 1-27. 
30. Schéle, E., et al., The gut microbiota reduces leptin sensitivity and the expression of the obesitysuppressing neuropeptides proglucagon (Gcg) and brain-derived neurotrophic factor (Bdnf) in the central nervous system. Endocrinology, 2013. 154(10): p. 3643-51.

31. Silva, Y.P., A. Bernardi, and R.L. Frozza, The Role of Short-Chain Fatty Acids From Gut Microbiota in Gut-Brain Communication. Front Endocrinol (Lausanne), 2020. 11: p. 25.

32. Bocci, V., The neglected organ: bacterial flora has a crucial immunostimulatory role. Perspect Biol Med, 1992. 35(2): p. 251-60.

33. Barandouzi, Z.A., et al., Altered Composition of Gut Microbiota in Depression: A Systematic Review. Front Psychiatry, 2020. 11: p. 541.

34. Cheung, S.G., et al., Systematic Review of Gut Microbiota and Major Depression. Front Psychiatry, 2019. 10: p. 34.

35. Zhao, F., et al., Alterations of the Gut Microbiota in Hashimoto's Thyroiditis Patients. Thyroid, 2018. 28(2): p. 175-186.

36. Chen, J., et al., Multiple sclerosis patients have a distinct gut microbiota compared to healthy controls. Sci Rep, 2016. 6: p. 28484.

37. Bravo, J.A., et al., Ingestion of Lactobacillus strain regulates emotional behavior and central GABA receptor expression in a mouse via the vagus nerve. Proc Natl Acad Sci U S A, 2011. 108(38): p. 16050-5.

38. Beilharz, J.E., et al., The effect of short-term exposure to energy-matched diets enriched in fat or sugar on memory, gut microbiota and markers of brain inflammation and plasticity. Brain Behav Immun, 2016. 57: p. 304-313.

39. Kau, A.L., et al., Human nutrition, the gut microbiome and the immune system. Nature, 2011. 474(7351): p. 327-36.

40. Nell, S., S. Suerbaum, and C. Josenhans, The impact of the microbiota on the pathogenesis of IBD: lessons from mouse infection models. Nat Rev Microbiol, 2010. 8(8): p. 564-77.

41. Atarashi, K., et al., Th17 Cell Induction by Adhesion of Microbes to Intestinal Epithelial Cells. Cell, 2015. 163(2): p. 367-80.

42. Ang, Q.Y., et al., Ketogenic Diets Alter the Gut Microbiome Resulting in Decreased Intestinal Th17 Cells. Cell, 2020. 181(6): p. 1263-1275.e16.

43. Wilck, N., et al., Salt-responsive gut commensal modulates $T(H) 17$ axis and disease. Nature, 2017. 551(7682): p. 585-589.

44. Kiner, E., et al., Gut CD4(+) T cell phenotypes are a continuum molded by microbes, not by $T(H)$ archetypes. Nat Immunol, 2021. 22(2): p. 216-228.

45. Huang, Y., et al., Prevotella Induces the Production of Th17 Cells in the Colon of Mice. J Immunol Res, 2020. 2020: p. 9607328.

46. Ivanov, II, et al., Specific microbiota direct the differentiation of IL-17-producing T-helper cells in the mucosa of the small intestine. Cell Host Microbe, 2008. 4(4): p. 337-49. 
47. Ekmekciu, I., et al., Immune Responses to Broad-Spectrum Antibiotic Treatment and Fecal Microbiota Transplantation in Mice. Front Immunol, 2017. 8: p. 397.

48. Gu, C., L. Wu, and X. Li, IL-17 family: cytokines, receptors and signaling. Cytokine, 2013. 64(2): p. 47785.

49. Kostic, M., et al., IL-17 and glutamate excitotoxicity in the pathogenesis of multiple sclerosis. Scand J Immunol, 2014. 79(3): p. 181-6.

50. Kolbinger, F., et al., IL-17A and Multiple Sclerosis: Signaling Pathways, Producing Cells and Target Cells in the Central Nervous System. Curr Drug Targets, 2016. 17(16): p. 1882-1893.

51. Huo, W., et al., Imbalanced spinal infiltration of Th17/Treg cells contributes to bone cancer pain via promoting microglial activation. Brain Behav Immun, 2019. 79: p. 139-151.

52. Prinz, M., S. Jung, and J. Priller, Microglia Biology: One Century of Evolving Concepts. Cell, 2019. 179(2): p. 292-311.

53. Feng, X., et al., Microglia mediate postoperative hippocampal inflammation and cognitive decline in mice. JCl Insight, 2017. 2(7): p. e91229.

54. Desbonnet, L., et al., Gut microbiota depletion from early adolescence in mice: Implications for brain and behaviour. Brain Behav Immun, 2015. 48: p. 165-73.

55. Fröhlich, E.E., et al., Cognitive impairment by antibiotic-induced gut dysbiosis: Analysis of gut microbiota-brain communication. Brain Behav Immun, 2016. 56: p. 140-55.

56. Walters, J.L., et al., Sevoflurane exposure has minimal effect on cognitive function and does not alter microglial activation in adult monkeys. Neurotoxicology, 2019. 71: p. 159-167.

57. Flanigan, T.J., C.D. Law, and S.A. Ferguson, Minimal effects from a single exposure to sevoflurane in adult male and female Sprague-Dawley rats. Neurotoxicol Teratol, 2021. 84: p. 106955.

58. Satomoto, M., et al., Sevoflurane preconditioning ameliorates lipopolysaccharide-induced cognitive impairment in mice. Exp Anim, 2018. 67(2): p. 193-200.

\section{Figures}



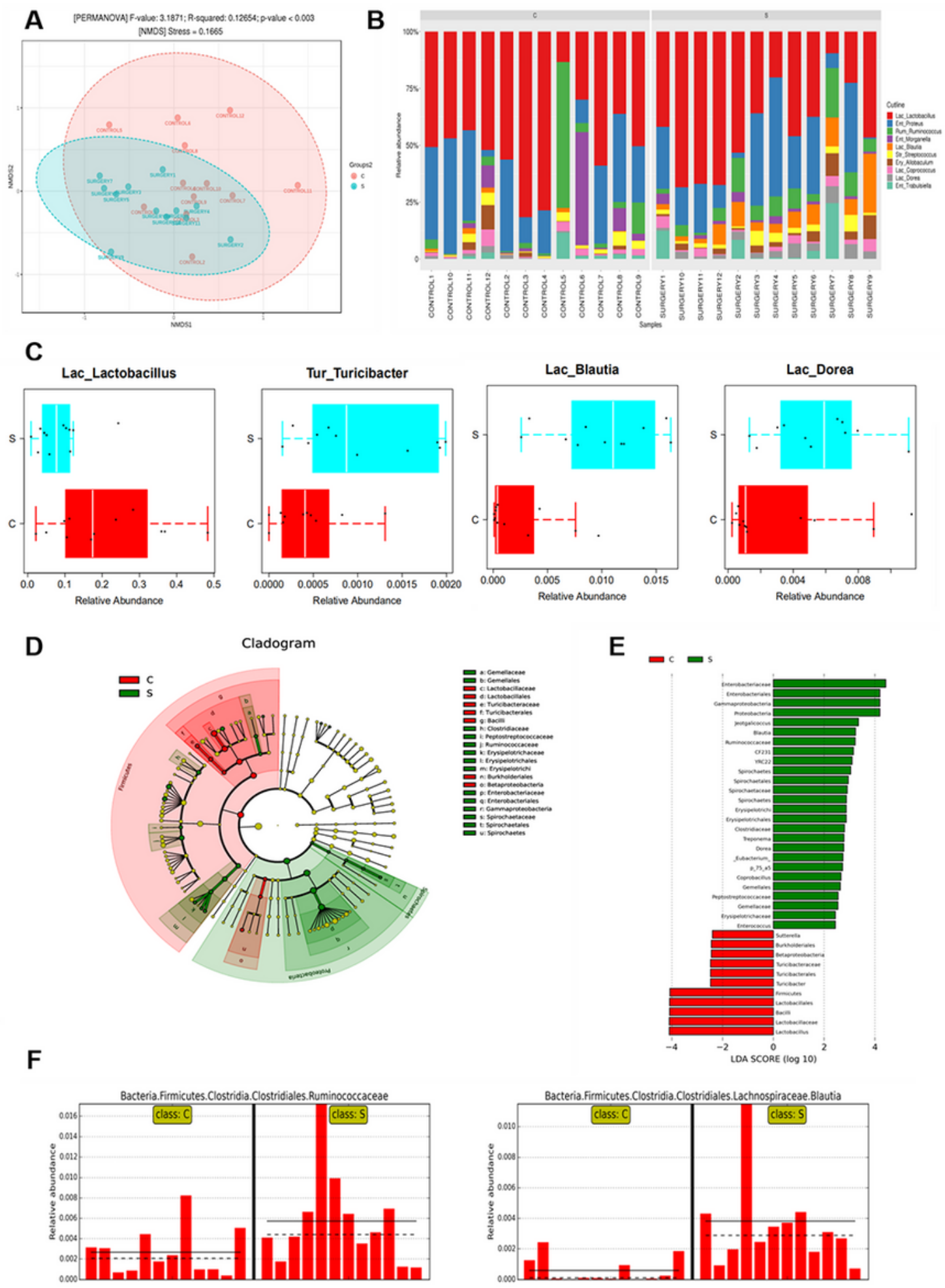

Figure 1

The difference of intestinal flora in the CON group and the SUR group. 


\begin{tabular}{c|c|c|c|c|c|c|}
\multicolumn{1}{c}{ A } & Antibiotics & $\begin{array}{c}\text { Anesthesia/ } \\
\text { surgery }\end{array}$ & OFT+YMT & FC Training & FC Test & Tissue harvest \\
\cline { 2 - 6 } & Day -28 & Day 0 & POD 3 & POD 4 & POD 5 & POD 6 \\
\hline
\end{tabular}

$\mathrm{n}=12$ per group

B

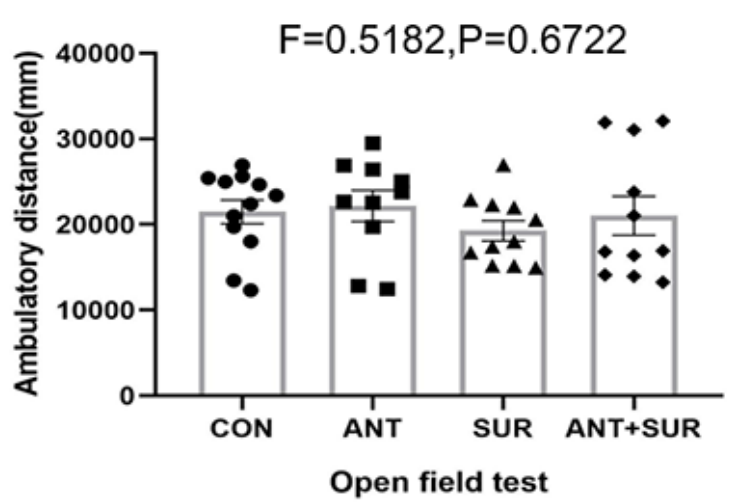

D

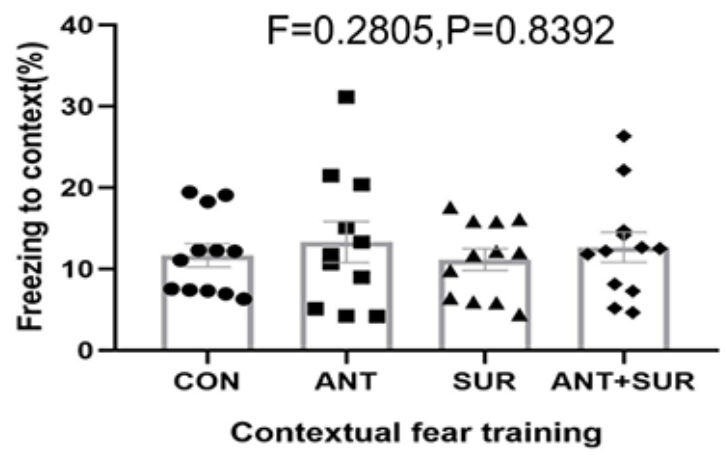

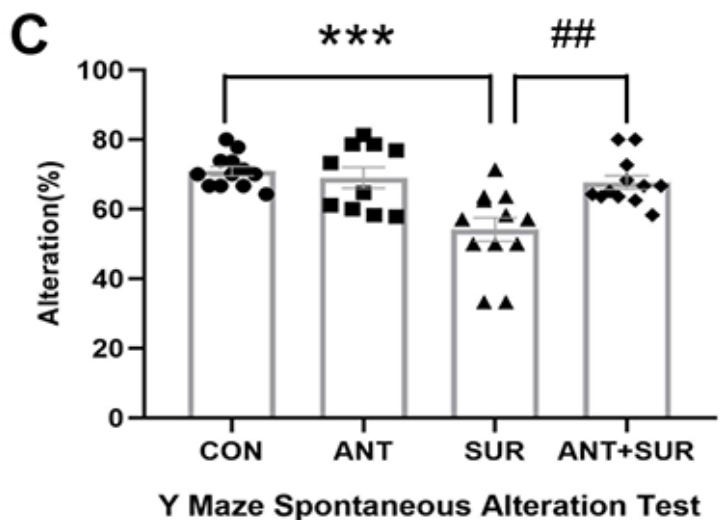

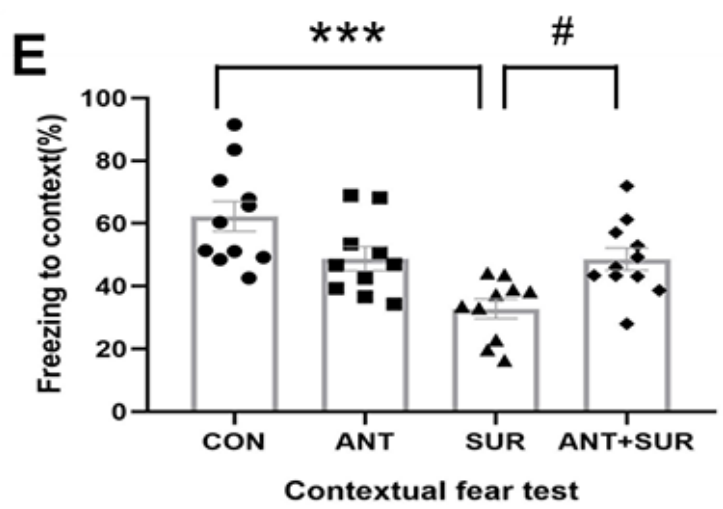

Figure 2

The administration of compound antibiotics before anesthesia/surgery improved postoperative cognitive function in aged rats. 

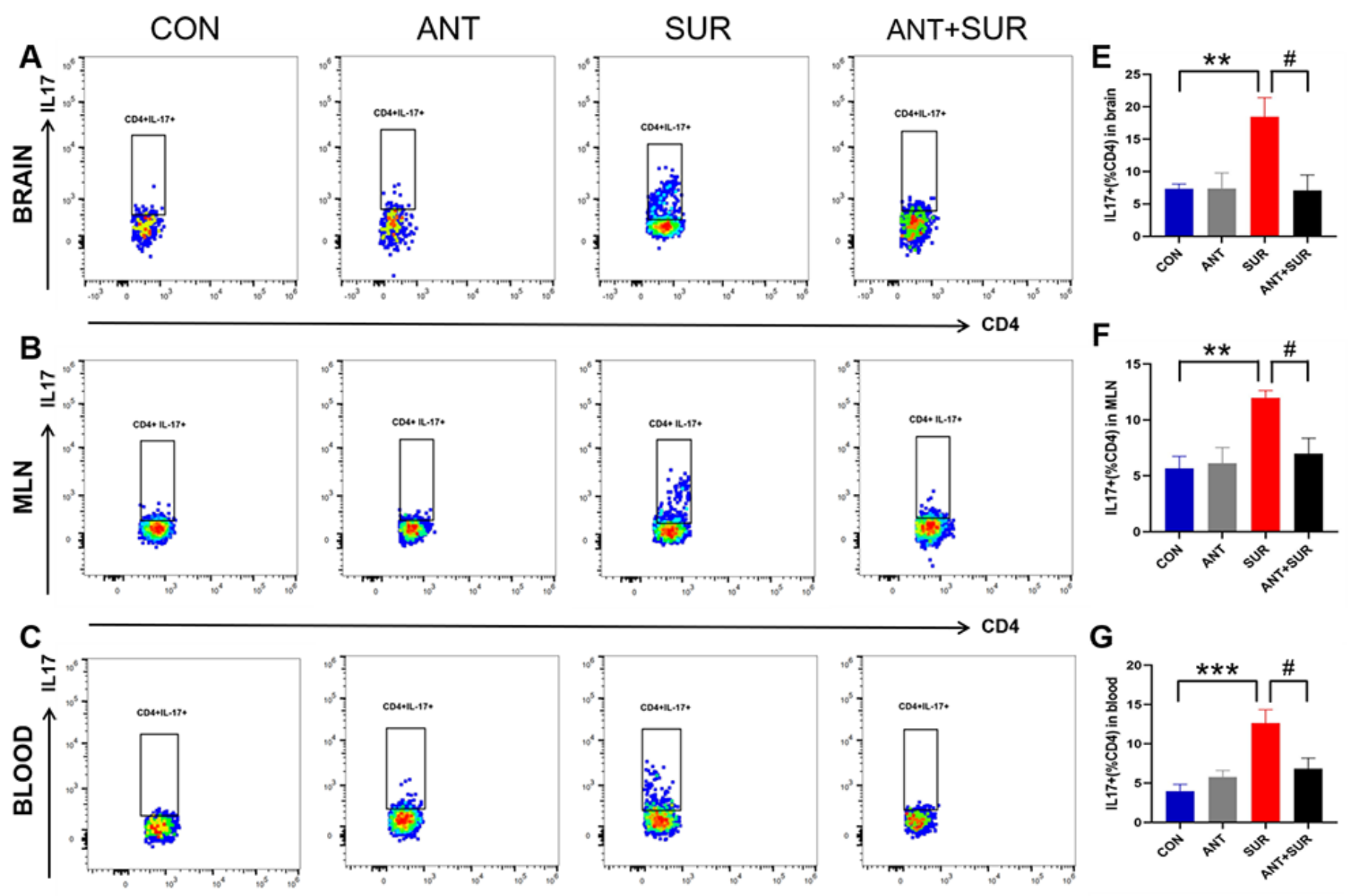

\section{G}
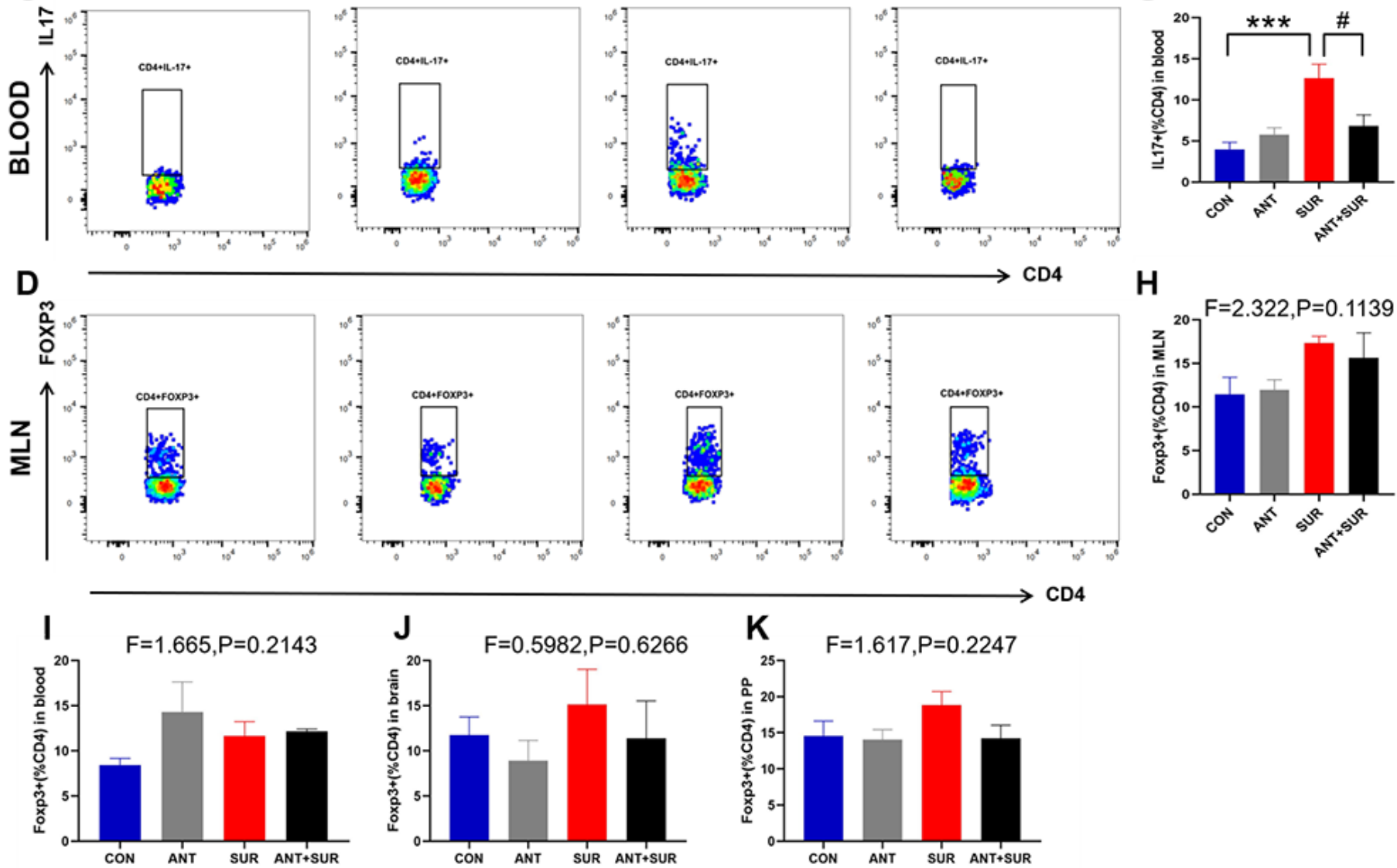

Figure 3

The number of Th17 cells and Foxp3 cells in the PP, MLN, blood and brain was detected by Flow cytometry. 


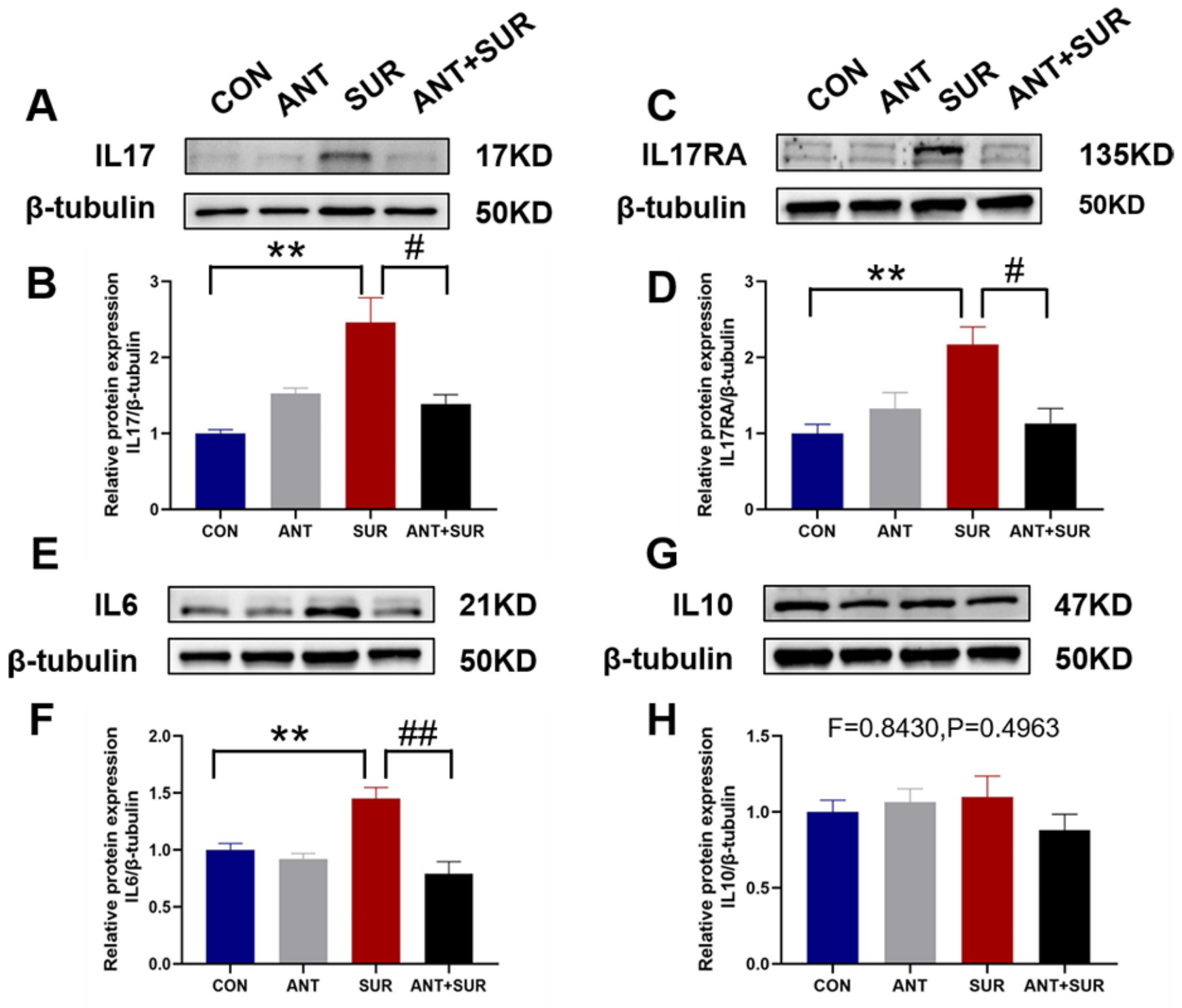

Figure 4

The Western blot results of inflammation-related proteins in the hippocampus at the sixth day after anesthesia/surgery. 

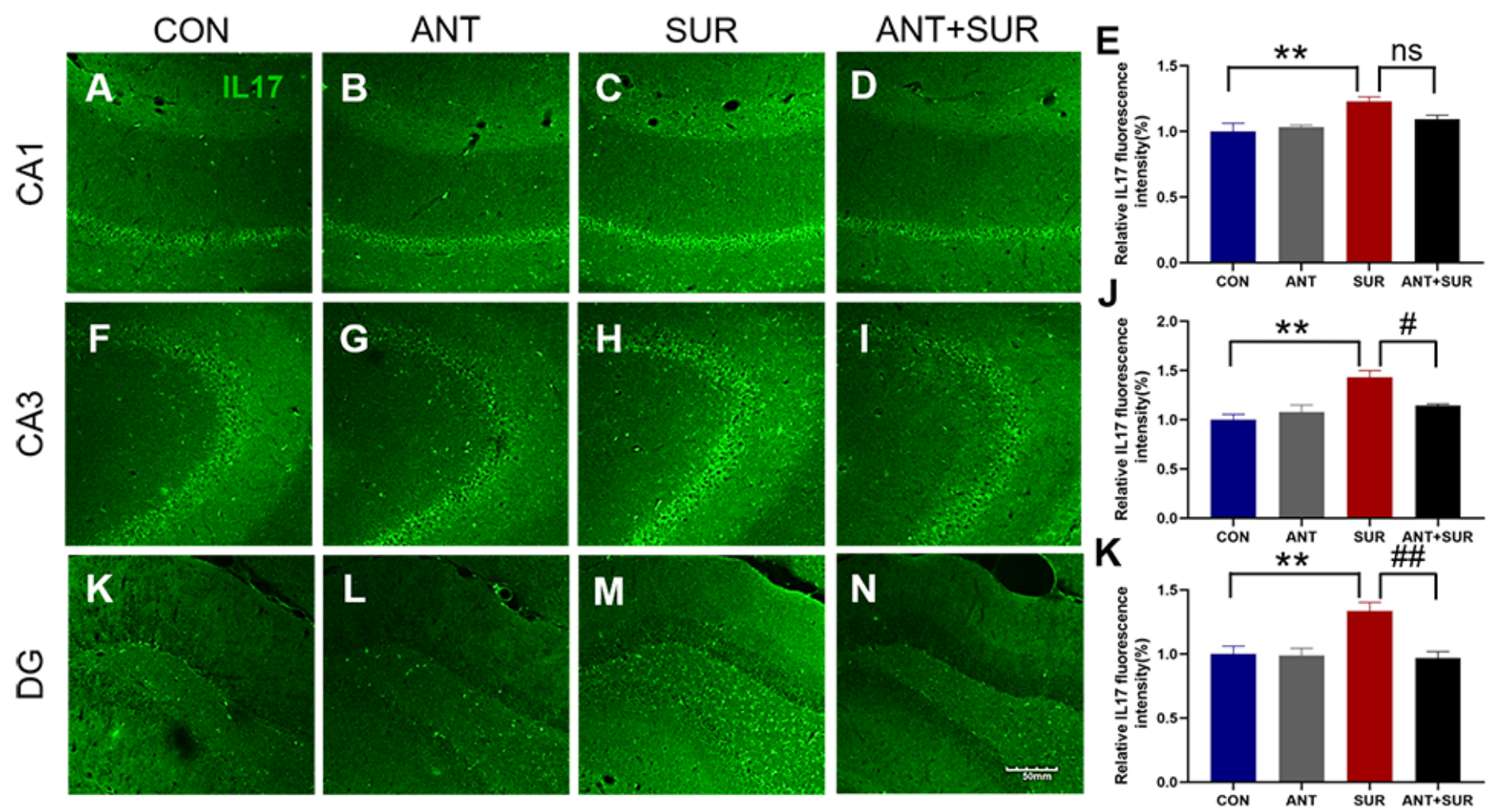

Figure 5

The immunofluorescence results of IL17 in the hippocampus at the sixth day after anesthesia/surgery. 


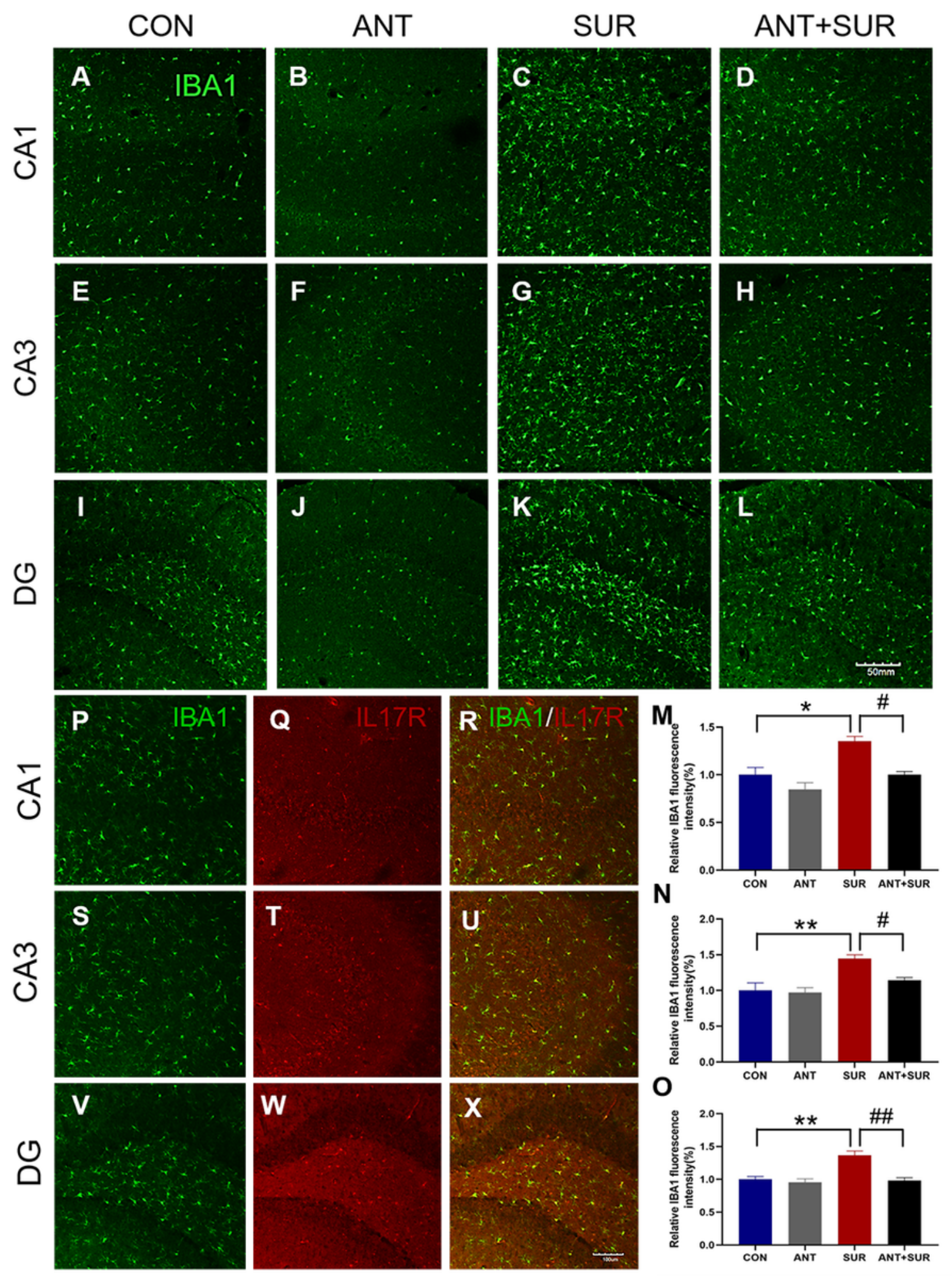

Figure 6

The immunofluorescence results of IL17R and IBA1 in the hippocampus at the sixth day after anesthesia/surgery 


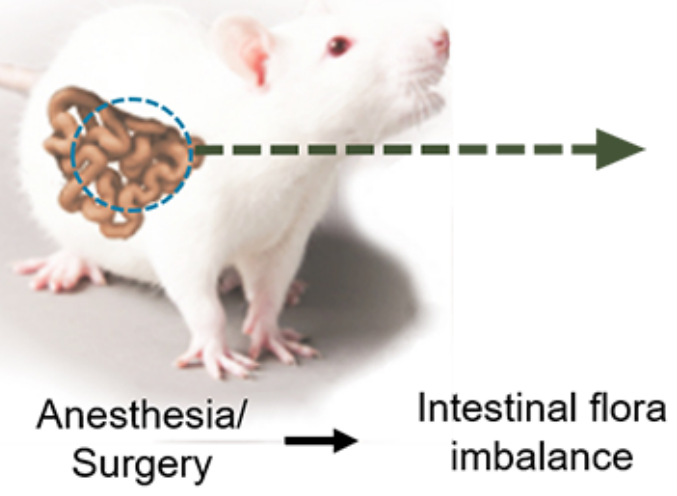

Cognitive impairment

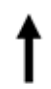

Activation of microglia $\longleftarrow$ dysfunction/leak

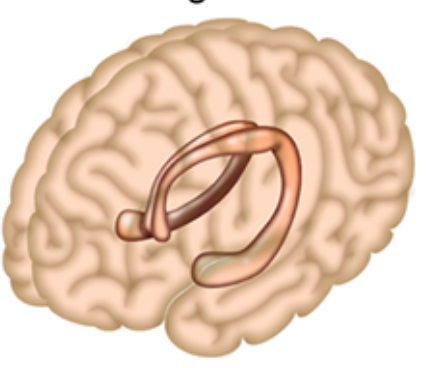

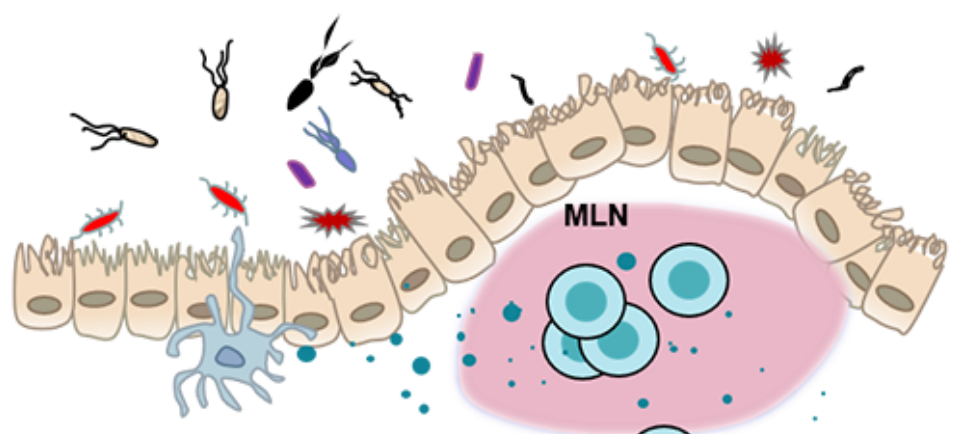

Activation

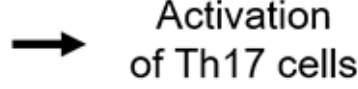

$\downarrow$ Increased IL17 and IL6

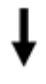

Circulatory

system

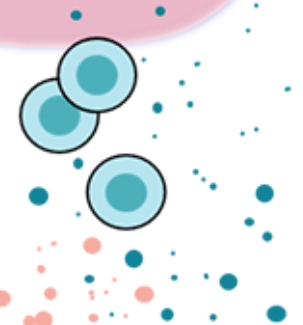

$\because \circ$

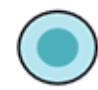

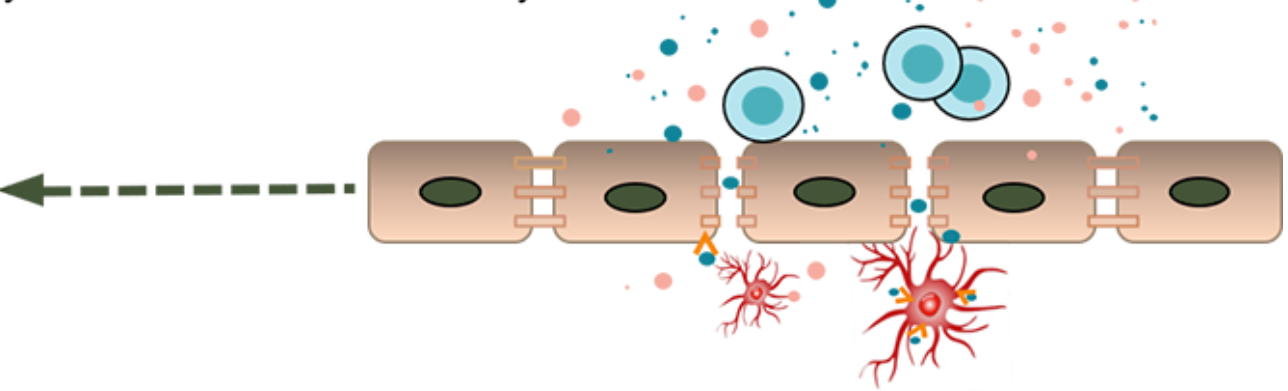

\section{Figure 7}

The mechanism diagram of this study.

\section{Supplementary Files}

This is a list of supplementary files associated with this preprint. Click to download.

- Additionalfile1.pdf

- Additionalfile2.pdf

- Additionalfile3.pdf

- Additionalfile4.pdf 